\title{
Activity Testing Model for Automatic Correction of Hand Pointing
}

Yalin Song $^{\mathrm{a}, \mathrm{b}}$, Yaoru Sun ${ }^{\mathrm{a},{ }^{*}}$, Hong Zhang ${ }^{\mathrm{a}, \mathrm{c}}$, Fang Wang ${ }^{\mathrm{d}}$

${ }^{\text {a }}$ Department of Computer Science and Technology, Tongji University, Shanghai, China

${ }^{\mathrm{b}}$ Complex Intelligent Network Institute, Henan University, Kaifeng Henan, China

${ }^{\mathrm{c}}$ Department of Mathematics, Taiyuan Normal University, Taiyuan Shanxi, China

${ }^{\mathrm{d}}$ Department of Computer Science, Brunel University, Uxbridge UB8 3PH, United Kingdom

${ }^{*}$ Corresponding author (yaoru@tongji.edu.cn)

\section{Abstract}

In this paper, an activity testing model was proposed to detect and assess automatic correction of hand pointing. The average recognition rate for automatic corrections of hand pointings was $98.2 \%$ using the acceleration data. Moreover, a score was calculated using the activity data of successful recognition and it provided sufficient estimation for the performance level of automatic correction. Experimental results showed that our model was effective and it could be applied to neurorehabilitation.

Key words: activity testing; automatic correction; hand pointing; rehabilitation

\section{Introduction}

Activity recognition can be used in the human-centric applications such as eldercare, healthcare and rehabilitation, especially the rehabilitation after brain injury or ischemia (e.g., stroke). Activity recognition has been widely investigated through accelerometer or wearable devices by many research groups [1-3]. Some daily activities, such as standing, walking, climbing up/down stairs or brushing teeth, have been analyzed with the classifiers. Ravi et al. [4] found that these activities can be recognized with fairly high accuracy using a single triaxial accelerometer. Hu el al. [5] and Yu et al. [6] investigated the pattern classification of surface electromyography (EMG) signals for activities of elbow extension and forearm pronation. In addition, some researchers $[7,8]$ studied activity recognition and applied this to the medical rehabilitation using the somatosensory devices, such as Nintendo Wii and Microsoft Kinect 3D sensor.

The automatic correction mechanism plays an important role in both planning and execution of visually guided movements in daily life $[9,10]$, and it can be a worthwhile method of neurorehabilitation. Although the research results of activity recognition had been plentiful, it is unclear whether automatic correction of hand pointing can be recognized accurately. 
In this work, an activity testing model of automatic correction was proposed to recognize and assess the performance of a certain group of hand pointings based on their trajectory signals recorded by a motion capture system. Two types of trajectory and acceleration data were extracted from the raw data of hand pointings. They were then processed and tested respectively using the same processing procedure. The correct classification data of hand pointings were calculated a score by the proposed scoring system to indicate the performance level of automatic correction of hand pointing.

The presented model has the following features: (1) It was based on the mechanism of automatic correction of hand pointing; (2) Two types of hand pointings were calculated and tested; (3) The score from the proposed scoring system provided sufficient estimation; (4) Our testing model can be applied to neurorehabilitation.

The rest of this paper is structured as follows: Section 2 reviews the related background studies, Section 3 presents the data processing and construction of the activity testing model, Section 4 shows the experimental results, and Section 5 concludes this work.

\section{Background}

The automatic correction mechanism allows human to quickly and involuntarily adjust ongoing hand movements (e.g., hand grasping and hand pointing) in response to the unexpected change of the target's properties (e.g., location). It is commonly a double-step hand pointing, namely, an initial pointing towards the first target location followed by a fast online correction to the final location $[9,10]$. Recent studies suggest that automatic corrections of hand pointings are mainly mediated by the dorsal visual pathway and associated with posterior parietal cortex (PPC) $[11,12,13]$. The neurological evidence supporting this view comes from the study on bilateral lesion of the PPC $[9,14,15]$, and transcranial magnetic stimulation (TMS) applied to the cortical areas to disrupt the unconscious correction [16]. In addition, the direct evidence of automatic correction in stereoscopic depth has been reported in our recent work [13].

Patients with brain injury (e.g., stroke) often suffer from hemiparesis and experience dramatic limitations in performing everyday activities $[17,18]$ (e.g., losing arm and hand movement skills). Therefore, it is very important to continue rehabilitation until maximum recovery has been achieved. We suggest that the rehabilitation based on automatic correction mechanism is worthwhile for patients with brain injury in PPC, and the method will help patients to relearn 
sensori-motor capabilities by exploiting the plasticity of the neuromuscular system. Virtual Reality (VR) based rehabilitation is an effective therapy which can help to improve patient motivation and sufficiently stimulate brain to remodel itself to provide better motor control and reduce therapy costs [19-21]. Chang et al. [20] and González-Ortega et al. [21] presented and assessed the intervention application based on Kinect device during the rehabilitation training. They found that the low-cost consumer game (Kinect-based) system could overcome the shortcomings of previous 2D systems because of using depth information and its motion tracking performance was satisfied to take the simple rehabilitation treatment. However, Kinect sensor has its drawback to be used as a tracking tool for automatic correction of hand pointing because of its poor frame rate (30fps). Although there have been studies which used optical motion capture system for activity analysis on individuals with neurological injury [19], the rehabilitation system based on automatic correction of hand pointing has not been reported yet.

In this work, we present that the automatic correction training of hand pointing can be as the effective therapy for upper limb motor rehabilitation to remodel brain areas after brain injury. The proposed data processing steps and scoring system of performance can assess the performance level of automatic correction of hand pointing. This score of hand pointing as the real-time feedback information further instructs patients to improve their automatic correction of hand pointing performance in a rehabilitation system.

\section{Design}

\subsection{Data Collection}

Hand pointing data from the motion capture system has the following attributes: time, coordinate in $\mathrm{X}$ axis (horizontal direction), coordinate in $\mathrm{Y}$ axis (upward height-direction) and coordinate in $\mathrm{Z}$ axis (depth direction). Participants sat in a dimly lit room with their chin resting on a chin-rest. Their eyes were $500 \mathrm{~mm}$ away from the monitor screen and aligned both vertically and horizontally with the center of the screen. The stimuli were presented by using a 3D LCD monitor (Zalman 3D, 22 inches, $1680 \times 1050$ pixels, $75 \mathrm{HZ}$ ), which was viewed through a polarized stereoscopic 3D spectacles (passive glasses with no receivers and no batteries). The positions of a participant's index finger wearing a marker (Infrared-emitting Diode, Maximum Frame Rate: 4600 $\mathrm{Hz}$ ) were recorded by the Optotrak Certus motion capture system (Maximum resolution: $0.01 \mathrm{~mm}$ ) with a temporal frequency of $200 \mathrm{~Hz}$. 
In the experiment, the classic double-step paradigm $[9,11,12]$ which instructs participant fast adjust ongoing hand pointing in response to the unexpected change (i.e., $20 \%$ change rate) of the target's location was adopted. The hand pointings were performed by thirteen participants. Each of them made 200 hand pointings (i.e., 200 trials), and was asked to reach and point to the target in $3 \mathrm{D}$ environment as quickly and accurately as possible within a limited time window ( $\leq 300 \mathrm{~ms})$. In each trial, a virtual circular target randomly appeared in one of the three depth positions, which were located at distances of $320 \mathrm{~mm}(\mathrm{~d} 1), 360 \mathrm{~mm}(\mathrm{~d} 2)$, and $400 \mathrm{~mm}(\mathrm{~d} 3)$ from the viewer respectively. In $20 \%$ of the trials, the target changed its depth position at the hand pointing onset and these trials were called the jump trials in which participants were asked to point to the perceived position and correct their index fingers to point to the new target position (i.e., automatic correction of hand pointing). The target jumped from $\mathrm{d} 1$ to $\mathrm{d} 2$ in half of the jump trials, and from $\mathrm{d} 2$ to $\mathrm{d} 3$ in the other half. The remaining 160 trials were called the static trials in which the target stayed in its initial position.

These trajectory data of the static and jump trials in 3D space were extracted from the raw data recorded by the motion capture system. The acceleration data along $\mathrm{X}$ axis, $\mathrm{Y}$ axis and $\mathrm{Z}$ axis were also calculated using these raw data. Figure 1 shows the sample of trajectory data for static trials and jump trials (i.e., automatic correction of hand pointing) in 3D space. Figure 2 shows the sample of the acceleration curves in three spatial orthogonal axes in the activities.

\subsection{Data Processing}

In order to build an excellent model of activity testing for automatic correction, two types of trajectory data and acceleration data were processed and tested respectively using the same processing procedure which consists of three steps: preprocessing, feature computation and classification.

\subsubsection{Preprocessing}

Because the lengths and the amplitudes of acceleration data were not equal for every hand pointings, we need the preprocessing to normalize these data before analysis. The preprocessing step comprises three sub-steps: denoising, normalization and resampling.

(1) Denoising

The obtained acceleration data contained measure noises and participants' unintended hand tremblings. It is necessary to get rid of such noises for extracting reliable features. A 1-D Gaussian 
smoothing was used to reduce the noises.

(2) Normalization

Given that the signal size of hand pointing changed according to the pointing force, the amplitudes of acceleration data were different between the hand activities. Normalization is a process for reducing this variation. In our work, the amplitudes for each axis' data were normalized to the interval $[-1,1]$ in all of the data. The normalized data are given as follows:

$$
K_{i}=L_{d}+\frac{\left(L_{u}-L_{d}\right) \times\left(P_{i}-\operatorname{Min}\right)}{\operatorname{Max}-\operatorname{Min}}
$$

where $P_{i}$ was the input data points, $L_{d}$ and $L_{u}$ were the boundary value of the interval [-1,1] respectively.

(3) Resampling

Because the raw data of hand pointings were sampled in the equal-time intervals $(5 \mathrm{~ms})$, the fast pointing interval had a small number of points and vice versa. Therefore, each of the acceleration data was resampled to the same length space. The predetermined unit length of these data was determined through experiment. The cubic spline interpolation was used to resample the acceleration data. In addition, zoom rates of resampling were calculated and saved to make the scoring system of our model.

\subsubsection{Feature Computation}

Extracting features is a fairly effective way to preserve class separability and can represent the characteristics of different activity signals in each hand pointing. Features' mean $(M)$, standard deviation $(S D)$, energy $(E)$, correlation between axes (Corr) and autoregressive coefficient model $(A R)$ were combined as a feature-type set $F_{t}$ to describe a single hand pointing. The form of the $F_{t}$ can be given by

$$
F_{t}=\{M, S D, E, \operatorname{Corr}, A R\}
$$

The mean $(M)$ feature is the DC component of the frequency domain over the frame of hand pointing. Standard deviation $(S D)$ of a hand pointing indicates the amplitude variability of a hand pointing.

The energy $(E)$ feature to capture data periodicity was used to discriminate automatic correction of hand pointings. The discrete Fourier transform (DFT) on each hand point data $Y(k)$ is obtained first by: 


$$
Y(k)=\operatorname{DFT}[x(n)]=\sum_{n=0}^{N-1} x(n) W_{N}^{n k} \quad 0 \leq k \leq N-1
$$

$11=\sum_{i=0}^{N-1} y_{i} \cdot z_{i}-\bar{y}$

$22+\sum_{j=1}^{p} a_{j} \cdot y(i-j)$ data after resampling.

$$
E=\frac{\sum_{n=0}^{N-1}\left|Y(i)^{2}\right|}{N}
$$

$$
\cdot \bar{Z}
$$
respectively.

$$
=e(i)
$$

Where $W_{N}$ is a periodic function and can be given as: $W_{N}=e^{-j \frac{2 \pi}{N}} . N$ is the length of activity

The energy (E) is

$$
i=\{1,2 \ldots N-1\}
$$

The correlation between axes is especially useful to discriminate the two-type hand pointings that involve translation in just one dimension. Only the correlation between $\mathrm{Y}$ axis and $\mathrm{Z}$ axis was calculated according to the motion characteristics of automatic correction of hand pointings. The covariance $\operatorname{cov}$ between the two axes of $\mathrm{Y}$ and $\mathrm{Z}$ can be given by:

where $\bar{y}$ and $\bar{z}$ are the mean value of the acceleration data in y axis and $\mathrm{z}$ axis respectively. A correlation coefficient $\operatorname{Corr}$ between axes can be given as:

$$
\operatorname{Corr}=\frac{\operatorname{cov}(y, z)}{S D_{y} \times S D_{z}}
$$

where $S D_{y}$ and $S D_{z}$ are the standard deviations of a hand pointing data in y axis and z axis

We used AR model to describe acceleration features of hand pointings due to the fact that short duration acceleration data can indeed be a kind of stationary random signal. The following AR model $\operatorname{AR}(p)$ is established for each acceleration component $y(i)$ :

4 where $a_{j}(j=1,2, \ldots, p), p$ are the model parameters to indicate the model order of the AR model, $e(i)$ is a white-noises sequence. Here the 4th-order AR coefficients were extracted from each of the three axes of the accelerometer data. 


\subsubsection{SVM-Based Classification}

Activity data for hand pointings included two classes of "static" and "jump" data and we used the support vector machine (SVM) to classify these data due to the fact that SVM is well known for its high recognition performance in binary classes [4]. SVM is a small sample size method based on statistic learning theory and has become one of the most popular classification methods in Machine Learning field in recent years. It is originally designed for binary classification to aim at finding the maximum-margin hyperplane using a transformation that maps the data from input space to feature space.

The feature-type sets $\left(F_{t}\right)$ were calculated as the input features of the SVM classifier to train and test. The data of "jump" and "static" classes had the same number of samples and they were operated five times, consistent with the previous studies [4-6]. The $80 \%$ samples of these two classes were randomly selected to train the SVM classifier and the remains were used to test. The classification result was the average of the five testing results.

Similarly, another type of trajectory data of hand pointings were operated by the same steps. The SVM classifiers were trained and tested using the two types of data, and the data type with the best recognition rate was selected to build activity testing model for automatic correction of hand pointing.

\subsection{Model Construction}

The activity testing model was shown in Figure 3. The model needed to generate one SVM classifier through training features (i.e., feature-type set $F_{t}$ ) of hand pointings. Moreover, the data of hand pointing recognized as the jump class (i.e., automatic correction of hand pointing) would generate a score by the scoring system of the model to indicate the performance level of automatic correction.

A centesimal grade $\mathrm{S}$ based on sectional normalization was adopted in the scoring system in which the critical values of sectional normalization were suggested in previous studies and our results on automatic correction of hand pointing $[9,13]$, and given by:

$$
\mathrm{S}=
$$




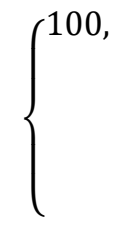

0 ,

$$
\begin{gathered}
L n_{d}+\frac{\left(L n_{u}-L n_{d}\right) \times\left(R_{i}-1\right)}{R m-1}, \\
L p_{d}+\frac{\left(L p_{u}-L p_{d}\right) \times\left(R_{i}-R m\right)}{R p-R m}, \\
R m \leq R_{i} \leq R p, L p_{d}=60, L p_{u}=1 \\
R_{i}>R m \text { or Failed Recognition }
\end{gathered}
$$

Where $R i$ was the zoom rate of input data and its length was $L i$. $L n$ was the length of activity data after resampling. LnMax and LpMax were the maximum lengths of activity data of automatic correction for normal persons and for patients respectively. $L n$ was assigned to the mean length occur within the specific time windows (LnMax) for normal persons. Similarly, $L p_{d}$ and $l p_{u}$ were more than LnMax and less than LpMax for patients. $R m$ and $R p$ were the zoom rates of normal persons and patients respectively. To determine LnMax, we calculated Z-scores [9] using $M L$ and standard deviation $(S D)$ of activity data for automatic correction in all jump trials. LnMax was the value with a length corresponding to a Z-score larger than 1.96 (i.e., $p=0.05$ two-tailed), namely, LnMax $=M L+1.96 \times S D$. As suggested by previous study on automatic correction of hand pointing for patients [9], LpMax was assigned to $500(\mathrm{~ms})$ here.

The score of performance indicates the successful automatic correction of hand pointing if it is more than 60 . The score suggests unsuccessful automatic correction if it is less than or equal 60 . automatic correction of next hand pointing as well as possible

\section{Experiment Results}

In our experiment, the behavioral data analysis clearly indicated that automatic correction evoked by depth could elicit fast corrective pointing movements before participants were aware of their 
intentional modifications. Our results showed that automatic correction was not affected by the target depth using repeated measures analysis of variance (ANOVA) [13]. Moreover, automatic correction of hand pointing in response to a depth jump could occur as early as within 190ms and the average duration of full hand pointings for automatic correction was $280 \mathrm{~ms}$, namely, $L n$ could be assigned to $280 \mathrm{~ms}$.

According to the results of our behavioral data analysis, the 190 samples of activity data derived from the correctly completed automatic correction in the jump trials and the same number of activity data in the static trials were extracted from the raw data recorded by the motion capture system. Trajectory and acceleration information in three spatial orthogonal axes were calculated from these data to test the recognition performance of SVM respectively. In each of types (trajectory data and acceleration data), 304 samples (i.e., $80 \%$ of total samples) of the two classes ("static" and "jump") were randomly selected to train the SVM classifier and the remains were used to test. These data of the two types (trajectory data and acceleration data) were both operated five times using SVM classifier. The classification result was the average of the five testing results (Table 1). The average recognition rate of the trajectory data was $83.4 \%$ and the acceleration data was further enhanced into $98.2 \%$. One possible reason for this difference is that the trajectory data included some redundant properties that would reduce the recognition rate but the acceleration data would not. Therefore, here the acceleration data were selected to build the activity testing model due to its high recognition rate.

\section{Conclusion}

In this work, we presented an activity testing model for automatic correction of hand pointing using acceleration data. The trajectory data and acceleration data were extracted from the raw data of hand pointings recorded by a motion capture system. The two type data of hand pointings were processed and tested respectively by using the same processing procedure that consisted of the data pre-processing, feature computation and classification. The average recognition rate for automatic correction of hand pointings was $98.2 \%$ using the acceleration data, which was better than using the traditional trajectory data. The score from our proposed scoring system using the activity data of successful recognition provided sufficient estimation for the performance level of automatic correction. Our results suggested that the activity testing model of automatic correction of hand pointing can be effective for the activity recognition of automatic correction of hand 
1 pointing.

\section{Acknowledgments}

This work was supported by the Grants from the National Natural Science Foundation of China (61173116), the National Science and Technology Pillar Program of China (2015BAF10B01), and the Science and Technology Commission of Shanghai Municipality (14JC1402203).

\section{References}

[1] T. Huynh, B. Schiele, Analyzing features for activity recognition, in: Proceedings of the 2005 joint conference on Smart objects and ambient intelligence: innovative context-aware services: usages and technologies, ACM, 2005, pp. 159-163.

[2] H. Ketabdar, P. Moghadam, M. Roshandel M, Pingu: A New Miniature Wearable Device for Ubiquitous Computing Environments, in: Sixth International Conference on Complex, Intelligent, and Software Intensive Systems. IEEE Computer Society, 2012, pp. 502-506.

[3] N. Sazonova, R. Browning, E. Melanson, E. Sazonov, E, Posture and activity recognition and energy expenditure prediction in a wearable platform, in: 36th Annual International Conference of the IEEE Engineering in Medicine and Biology Society. IEEE, 2014, pp. 4163-4167.

[4] N. Ravi, N. Dandekar, P. Mysore, M.L. Littman, Activity recognition from accelerometer data, in: AAAI, 2005, pp. 1541-1546.

[5] X. Hu, V. Nenov, Multivariate AR modeling of electromyography for the classification of upper arm movements, Clinical neurophysiology, 115 (6) (2004) 1276-1287.

[6] W. Yu, H. Yamaguchi, H. Yokoi, M. Maruishi, Y. Mano, Y. Kakazu, EMG automatic switch for FES control for hemiplegics using artificial neural network, Robotics and Autonomous Systems, 40 (2) (2002) 213-224.

[7] G. Saposnik, R. Teasell, M. Mamdani, J. Hall, W. McIlroy, D. Cheung, K.E. Thorpe, L.G. Cohen, M. Bayley, Effectiveness of virtual reality using Wii gaming technology in stroke rehabilitation a pilot randomized clinical trial and proof of principle, Stroke, 41 (7) (2010) $1477-1484$

[8] R.A. Clark, Y.-H. Pua, K. Fortin, C. Ritchie, K.E. Webster, L. Denehy, A.L. Bryant, Validity 
of the Microsoft Kinect for assessment of postural control, Gait \& posture, 36 (3) (2012) $372-377$.

[9] L. Pisella, H. Grea, C. Tilikete, A. Vighetto, M. Desmurget, G. Rode, D. Boisson, Y. Rossetti, An 'automatic pilot' for the hand in human posterior parietal cortex: toward reinterpreting optic ataxia, Nature neuroscience, 3 (7) (2000) 729-736.

[10] H. Johnson, R.J. van Beers, P. Haggard, Action and awareness in pointing tasks, Experimental brain research, 146 (4) (2002) 451-459.

[11] R. D. McIntosh, A. Mulroue, J. R. Brockmole, How automatic is the hand's automatic pilot? Experimental Brain Research, 206 (3) (2010) 257-269.

[12] L. O.Wijdenes, E. Brenner, J. B. Smeets, Fast and fine-tuned corrections when the target of a hand movement is displaced, Experimental Brain Research, 214 (3) (2011) 453-462.

[13] Y. Song, Y. Sun, J. Zeng, F. Wang, Automatic Correction of Hand Pointing in Stereoscopic Depth, Scientific reports, 4 (2014) 7444.

[14] A. Blangero, V. Gaveau, J. Luaute, G. Rode, R. Salemme, M. Guinard, D. Boisson, Y. Rossetti, L. Pisella, A hand and a field effect in on-line motor control in unilateral optic ataxia, Cortex, 44 (5) (2008) 560-568.

[15] I. T. Mahayana, L. Tcheang, C. Chen, C. Juan, N. G. Muggleton, Posterior parietal cortex and visuospatial control in near and far space, Translational Neuroscience, 5 (4) (2014) 269-274.

[16] M. Desmurget, C. Epstein, R. Turner, C. Prablanc, G. Alexander, S. Grafton, Role of the posterior parietal cortex in updating reaching movements to a visual target, Nature neuroscience, 2 (6) (1999) 563-567.

[17] M. Simonetta-Moreau, Non-invasive brain stimulation (nibs) and motor recovery after stroke. Annals of Physical \& Rehabilitation Medicine, 57(8) (2014) 530-542.

[18] R. Teasell, Stroke recovery and rehabilitation, Stroke, 34 (2) (2003) 365-366.

[19] A. Mirelman, B. L. Patritti, P. Bonato, J. E. Deutsch, J. E, Effects of virtual reality training on gait biomechanics of individuals post-stroke, Gait \& Posture, 31(4) (2010) 433-437.

[20] C.Y. Chang, B. Lange, M. Zhang, S. Koenig, P. Requejo, N. Somboon, A.A. Sawchuk, A.A. Rizzo, Towards pervasive physical rehabilitation using Microsoft Kinect, in: Pervasive Computing Technologies for Healthcare (PervasiveHealth), 2012 6th International Conference on, IEEE, 2012, pp. 159-162. 
1 [21] D. González-Ortega, F. J. Díaz-Pernas, M. Martínez-Zarzuela, M. Antón-Rodríguez, A 2 Kinect-based system for cognitive rehabilitation exercises monitoring, Computer Methods \& Programs in Biomedicine, 113(2) (2013) 620-631.

4 


\section{$1 \quad$ Figure Legends}

2 Figure 1. The sample of trajectory data. (A) for the target jumped from d1 to $\mathrm{d} 2 \mathrm{in}$ the jump trials. (B) for the 3 target jumped from $\mathrm{d} 2$ to $\mathrm{d} 3$ in the jump trials. (C) and (D) for the static trials at depth "d1"and depth "d2".

4 Figure 2. The sample of acceleration data. (A) for the target jumped from d1 to d2 in the jump trials. (B) for the 5 target jumped from $\mathrm{d} 2$ to $\mathrm{d} 3$ in the jump trials. (C) and (D) for the static trials at depth "d1"and depth "d2".

6 Figure 3. Activity testing model scheme for automatic correction of hand pointing.

7

8

9 
1 Tables

2

Table 1. Recognition results of the trajectory and acceleration data

\begin{tabular}{ccc}
\hline \multirow{2}{*}{ Times } & \multicolumn{2}{c}{ Recognition rate (\%) } \\
\cline { 2 - 3 } & Trajectory Data & Acceleration Data \\
\hline 1 & $76.32 \%$ & $96.05 \%$ \\
3 & $85.53 \%$ & $97.37 \%$ \\
4 & $81.58 \%$ & $100 \%$ \\
5 & $92.11 \%$ & $98.68 \%$ \\
\hline Average accuracy & $81.58 \%$ & $98.68 \%$ \\
\hline
\end{tabular}

3 